\title{
Integration of Blockchain in Internet of Things
}

\author{
Tri A. Sundara ${ }^{1}$ and Lathifah Arief ${ }^{2}$
}

\begin{abstract}
Internet of Things (IoT) enables services by interconnecting (physical and virtual) things and integrate it into communication networks. On the other side, Blockchain enable us to have a distributed peer-to-peer network where non-trusting members can interact with each other without a trusted intermediary. This research aims to explore the potential integration of Blockchain in IoT.
\end{abstract}

Keywords - internet of things (IoT), blockchain.

\section{INTRODUCTION}

$I_{\text {in }}^{\mathrm{N}}$ NTERNET of Things may lead to a hyper-connected future in which every- thing is connected to everything else appears. Estimates for the number of connected devices range from 25 to 50 billion by the year 2020. [1] This promise seems to draw closer and closer. Other development in related field also draw many attentions in recent years. Blockchain, the distributed ledger technology, enables a trust-less network. Initially conceived as a peer-to-peer cash payment system, it has been used in many other areas as well. [2] The two has been seen like Internet, which initially used in limited fields, but now seems omnipresence in everyday live. [3].

\section{INTEGRATION OF BLOCKCHAIN IN INTERNET OF THINGS} (IOT)

\section{A. Internet of Things}

IoT as "a global infrastructure for the information society, enabling advanced services by interconnecting (physical and virtual) things based on existing and evolving interoperable information and communication technologies" and for these purposes a "thing" is "an object of the physical world (physical things) or the information world (virtual things), which is capable of being identified and integrated into communication networks". The IoT allows objects to be sensed or controlled remotely across existing network infrastructure, creating opportunities for more direct integration of the physical world into computer-based systems, and resulting in improved efficiency, accuracy and economic benefit in addition to reduced human intervention. When IoT is augmented with sensors and actuators, the technology becomes an instance of the more general class of cyber-physical systems, which also encompasses technologies such as smart grids, virtual power plants, smart homes, intelligent transportation and smart cities.

Tri A. Sundara is with the lecturer of Department of Information System, STMIK Indonesia

Lathifah Arief is with the Department of Computer System, Andalas University, Indonesia.
Each thing is uniquely identifiable through its embedded computing system but is able to interoperate within the existing Internet infrastructure.

\section{B. Blockchain}

A blockchain is a distributed database that is used to maintain a continuously growing list of records, called blocks. Each block contains a timestamp and a link to a previous block. A blockchain is typically managed by a peer-to-peer network collectively adhering to a protocol for validating new blocks. By design, blockchains are inherently resistant to modification of the data. Once recorded, the data in any given block cannot be altered retroactively without the alteration of all subsequent blocks and the collusion of the network. Functionally, a blockchain can serve as "an open, distributed ledger that can record transactions between two parties efficiently and in a verifiable and permanent way. The ledger itself can also be programmed to trigger transactions automatically."

\section{Integration of Blockchain in IoT}

Blockchain can benefit IoT in some ways [4]. The trust modelling in IoT could be leveraged by using Blockchain [5].

Many IoT applications involve regularly gathering small bits of data from edge nodes over long periods of time. The kinds of information involved are endless: valve settings, meter readings, environmental conditions like temperature and humidity, geolocation points, water levels, maintenance inspection updates, you name it. Naturally, this data is often uploaded to a time series database. Adding it to a blockchainbased ledger-which, after all, is essentially a cryptographically-assured time series database - makes perfect sense for many IoT applications.

A decentralized architecture for the ever-expanding IoT device ecosystem to be sustainable. From the manufacturer's side, the current centralized model has a high maintenance cost. consider the distribution of software updates to millions of devices for years after they have been long discontinued.

From the consumer's side, there is a justified lack of a trust in devices that "phone home" in the background and a need for a "security through transparency" approach. These issues can be solved with a scalable, trust-less peer-to-peer model that can operate transparently and distribute data securely; the authors correctly point out that a blockchain provides an elegant solution to this problem.

Consider the following setup to get an understanding of how this could work. All the IoT devices of a manufacturer operate on the same blockchain network. The manufacturer deploys a 
smart contract that allows them to store the hash of the latest firmware update on the network. The devices either ship with the smart contract's address baked into their blockchain client, or they find out about it via a discovery service. They can then query the contract, find out about the new firmware, and request it by its hash via a distributed peer-to-peer file system such as IPFS.

The first requests for this file will be served by the manufacturer's own node (also taking part into the network), but after the binary has propagated to enough nodes, the manufacturer's node can stop serving it. Assuming the devices are configured so as to share the binary they got a device that joins the network long after the manufacturer has stopped participating in it, can still retrieve the sought after firmware update and be assured that it is the right file. This all happens automatically, without any user interaction. Compare and contrast with the centralized scenario where the device polls the manufacturer's server for an update and gets a 404 error.

Furthermore, a blockchain network where crypto currency is exchanged provides a convenient billing layer and paves the way for a marketplace of services between devices. In the example above, devices that store a copy of the binary may charge for serving it, in order to sustain their infrastructure costs (or simply to make a profit). Other examples include:

All the IoT devices of a manufacturer operate on the same blockchain network. The manufacturer deploys a smart contract that allows them to store the hash of the latest firmware update on the network. The devices either ship with the smart contract's address baked into their blockchain client, or they find out about it via a discovery service.[7]

\section{D.Blockchain-IoT Combination Use-Cases}

These are some benefits of placing the resulting data in a blockchain-based ledger. Here are some examples:

- Operation of an oil refinery

Oil refinery might receive crude oil feedstock inputs from a number of well heads, send processed outputs via rail and truck, function as an authoritative measurement point for purposes of extraction taxes, involve the contributions of several different operations and maintenance companies for specialized sub-systems, and necessitate compliance with a large number of regulations. The opportunities for cost savings and process improvements are significant. Automated measurement of on-site and in-field flow meters through standardized sensors and IoT connectivity solutions can enable precise determination of production from each well head. By instrumenting a few hundred key maintenance points, the refinery operators can reduce the need for manual inspections by personnel walking around with clipboards (still surprisingly common even today).

\section{-Vehicles authentication}

Strong authentication of delivery vehicles at well heads and truck outs, along with remote monitoring of pressure drops, can help prevent theft of feedstock and refined materials, which is all too frequent in the industry.
- $\operatorname{Tax}$

Taxation authorities can trust production amounts down to the individual wellhead, leading to more accurate and efficient revenue allocation. In addition, the producers can eliminate expensive "production accounting" functions.

- Regulatory agencies can determine compliance based on assured data from instrumentation on site, instead of relying on post-hoc audits of a voluminous paper trail (which are often charged back to the operator).

- Insurance companies.

They can provide reduced rates because maintenance and safety records are authenticated and immutable.

- Multiple parties can have the same view on data reflecting joint operation and maintenance of the plant, thus increasing trust and improving coordination.

- Filecoin which allows devices to "rent their disk space", and EtherAPIs, which make it possible to monetize API calls the caller needs to provide the necessary micropayment (in Bitcoin or Ethereum respectively) before requesting them. With a cryptocurrency in place, every device can have its own bank account on the Internet; it can then expose its resources to other devices (or users) and get compensated for their usage via microtransactions.

- Sharing of services and property in general.

Smart electronic locks can be unlocked with a device that carries the appropriate token. These tokens are bought on blockchain. The owner of a smart lock that wishes to rent their house or car sets a price for timed access to that electronic door lock. An interested party can use a mobile app to identify the slock, pay the requested amount, then communicate with the lock via a properly signed message. Billing is simplified by having all the locks operating on the same blockchain.

- Chemical industry

Blockchain could be employed to facilitate machine- tomachine (M2M) interactions and establish a M2M electricity market in the context of the chemical industry.

Other similar improvements can be achieved in domains as diverse as transportation (e.g., automated tracking of intermodal containers), agriculture (e.g., chain of custody for perishables), mining (e.g., collision avoidance at open-pit mines), and manufacturing (e.g., inventory control and production reporting). Although such data would not necessarily be stored in the public blockchain for reasons of scalability, cost, and privacy, using a private blockchain that is shared among the relevant parties (e.g., within a supply chain) is an excellent alternative.

\section{CONCLUSION}

We may conclude that integrating blockchain in IoT can cause significant transformations across several industries, paving the way for new business models and novel, distributed applications. IoT devices could be used as sources to collect data in real-time or near real-time and Blockchain for secure access and data exchange between institutions. 


\section{ACKNOWLEDGMENT}

The authors would like to thank STMIK Indonesia and Andalas University for its generous support. This research is funded by 2017 grant from STMIK Indonesia..

\section{REFERENCES}

[1] T. Saarikko, U. H. Westergren, and T. Blomquist, "The Internet of Things : Are you ready for what 's coming ?,” Bus. Horiz., 2017.

[2] [2] S. Nakamoto, "Bitcoin: A Peer-to-Peer Electronic Cash System," pp. 1-9, 2008.

[3] [3] M. Swan, Blockchain: Blueprint for a New Economy. 2015.

[4] [4] S. Huckle, R. Bhattacharya, M. White, and N. Beloff, "Internet of Things, Blockchain and Shared Economy Applications," Procedia Comput. Sci., vol. 58, pp. 461-466, 2016.

[5] [5] C. Fernandez-gago, F. Moyano, and J. Lopez, "Modelling Trust Dynamics in the Internet of Things," Inf. Sci. (Ny)., 2017.

[6] [6] J. J. Sikorski, J. Haughton, and M. Kraft, "Blockchain technology in the chemical industry: Machine-to-machine electricity market," Appl. Energy, vol. 195, pp. 234-246, 2017.

[7] [7] K. Christidis and G. S. Member, "Blockchains and Smart Contracts for the Internet of Things," vol. 4, 2016. 